Key-words: Malaria. Toxoplasmosis. Amœbiasis. Leishmaniasis. Trypanosomiasis. Filariasis. Onchocerciasis. Schistosomiasis. Hydatidosis. Cysticercosis.

\title{
LES SONDES MOLÉCULAIRES DANS L'ÉTUDE ET LE DIAGNOSTIC DES MALADIES PARASITAIRES
}

\author{
P. AMBROISE-THOMAS
}

\begin{abstract}
RÉSUMÉ
Les sondes moléculaires ou les techniques qui en dérivent ont, en moins de 10 ans, très largement amélioré nos connaissances des maladies parasitaires. Indépendamment de la taxonomie, ces méthodes ont apporté des résultats très démonstratifs sur le plan diagnostique, épidémiologique ou pathogénique. Très sensibles et le plus souvent hautement spécifiques, les sondes moléculaires permettent, d'un point de vue diagnostique, de déceler la présence d'un très petit nombre de parasites. Cette détection est également valable quel que soit le stade parasitaire donné. Elle s'applique en particulier à l'identification de formes infestantes chez les hôtes vecteurs. L'identification spécifique d'espèces parasitaires pourtant voisines renseigne sur l'infectivité pour l'homme d'espèces
\end{abstract}

jusqu'ici réputées uniquement zoophiles. Enfin, en permettant l'identification spécifique de souches, d'isolats ou mêmes de clones particuliers, les sondes moléculaires révèlent des différences qui peuvent être rapportées à un pouvoir pathogène inégal, à certaines caractéristiques biologiques particulières, ou encore à des sensibilités différentes aux médicaments disponibles. Dans cette revue générale, en nous appuyant notamment sur notre expérience personnelle, nous rapportons les résultats principaux obtenus en matière de paludisme et de toxoplasmose, et également d'amibiase, de leishmanioses, de trypanosomoses, de filarioses lymphatiques, d'onchocercose, de bilharzioses, d'échinococcoses, et de cestodoses.

\section{SUMMARY: Molecular probes in the study and diagnosis of parasitic diseases.}

Molecular biology techniques have contributed, in the last ten years, to a better understanding of parasitic diseases. DNA probes, for example, have been successfully used not only for taxonomic purposes, but also for the diagnosis, the epidemiology and the pathogenicity of these infections. Due to their high sensitivity and specificity, the DNA probes allow, from a diagnostic point of view, the detection of wery few of parasites in a given sample. This detection is also valid for any parasitic stage considered. It can be specially used to detect the infective stage in the vector, which, in epidemiological studies, is very important. The iden- tification of parasitic species or sub-species indicates the human infectivity of species considered in the past as zoophilic. Finally, by allowing the specific identification of strains, isolates or even particular clones, the DNA probes also show differences which can be related to pathogenicity, to particular biological characteristics, or even to drug sensitivity. In this review, I relate the main results obtained in malaria and toxoplasmosis in our laboratory in Grenoble. I will also consider some recent data on amœbiasis, leishmaniasis, trypanosomiasis, filariasis, schistosomiasis and echinococcosis.
En quelques années, les sondes moléculaires et les techniques qui en dérivent, comme la réaction d'amplification génique in vitro (Polymerase Chain Reaction ou PCR) ont très largement amélioré nos connaissances de plusieurs maladies parasitaires. Souvent spectaculaires, les résultats sont en effet très sensibles et très spécifiques, ce qui s'explique par la nature même des sondes moléculaires qui mettent en évidence les fragments les plus caractéristiques du génome parasitaire. Le nombre de communications consacrées à

Département de Parasitologie-Mycologie Médicale et Moléculaire (DP3M), Université Joseph Fourier-Grenoble I, URA CNRS 1344. ces méthodes, dans le cadre d'ICOPA VII traduit bien l'intérêt de la question. Par ailleurs, les données bibliographiques sont déjà abondantes dans ce domaine et rapportent de nombreux résultats originaux. Nous tenterons d'en présenter une synthèse et, pour éviter le risque d'une énumération fastidieuse et inévitablement incomplète, nous nous limiterons délibérément aux plus importants de ces résultats en matière de diagnostic, d'épidémiologie, de pouvoir pathogène et de sensibilité au traitement. Pour le paludisme et pour la toxoplasmose, cette revue générale sera complétée par la présentation de résultats obtenus à Grenoble. Pour d'autres protozooses (amibiase, leishmanioses, trypanosomes africaines et américaines) ou pour des ver- 
minoses (filarioses lymphatiques, onchocercose, bilharzioses, échinococcoses et cestodoses), nous nous limiterons à la synthèse des données bibliographiques actuellement disponibles.

\section{Paludisme}

Parmi les quatre espèces plasmodiales parasites de l'homme, Plasmodium falciparum a été longtemps la seule pour laquelle des sondes moléculaires ont été développées. La plupart de ces sondes sont constituées de séquences répétées, appartenant à la famille de séquences identifiées par Franzen et al. (1984). Radiomarquées au ${ }^{32} \mathrm{P}$ ou couplées à une enzyme, ces sondes sont capables, dans des conditions expérimentales, de détecter 50 à 500 Plasmodium/ $\mu \mathrm{l}$ de sang. Au cours de plusieurs travaux sur le terrain réalisés en Thaillande, la valeur pratique de ces sondes (en particulier de l'oligonucléotide pFR 1) comme moyen de dépistage a pu être précisée et comparée à celles des autres moyens de détection. Dans ces conditions pratiques, la sensibilité paraît légèrement diminuer, mais la très grande spécificité est confirmée de même que l'intérêt dans le suivi parasitologique de chimio-résistances. Plus récemment, quatre sondes oligo-nucléotidiques ( $\mathrm{pFR} 1 \mathrm{AP}$, clone 26, pPS 14, et p 242 B) ont été utilisées pour déceler l'apparition et l'évolution de la parasitémie chez des patients expérimentalement infestés. Les résultats sont décevants puisque les sondes moléculaires ne détectent que 5 à $28 \%$ des parasitémies décelées par les autres méthodes (gouttes épaisses, cultures in vitro).

A Grenoble, nous avons pu cloner et séquencer (Oury et al.) une sonde (pUF 28) de Plasmodium falciparum, constituée de la répétition d'une séquence de 21 paires de bases (pb) en 87 copies qui diffèrent entre elles par des modifications de 1 à 9 nucléotides.

Cette sonde est étroitement spécifique de Plasmodium falciparum et, en hybridation sur nitrocellulose (Dot blot) elle révèle un fragment génomique commun à toutes les souches de ce parasite. En revanche, la sonde pU F 28 n'hybride pas l'ADN humain si l'ADN des trois autres espèces plasmodiales parasites de l'homme. Après radiomarquage par le ${ }^{32} \mathrm{P}$, la sonde pUF 28 détecte environ 50 Plasmodium $/ \mu$ l de sang, ce qui correspond à une parasitémie de $0,01 \%$ dans un échantillon de $50 \mu \mathrm{l}$. Cette sensibilité est sensiblement comparable à celle de la goutte épaisse $(20$ à 50 Plasmodium/ $\mu$ l) examinée dans les meilleures conditions et par des microscopistes entraînés. La technique a été par ailleurs améliorée pour être réalisable, en totalité, en $36 \mathrm{~h}$, et pour permettre l'analyse simultanée, chaque jour, de 100 échantillons sanguins environ. Plus récemment, un marquage «froid" par la digogénine a permis de mieux adapter pUF 28 aux conditions d'utilisation sur le terrain. Parallèlement, le test a été simplifié et raccourci de façon à ce que la réaction soit effectuée en totalité en 5 à 12 heures.
En pratique, il est évidemment indispensable de préciser l'intérêt de méthodes de diagnostic aussi sensibles. Dans les zones de transmission palustre à forte prévalence, une sensibilité élevée n'est évidemment pas nécessaire. En revanche, l'intérêt des sondes moléculaires est de permettre, pour des enquêtes épidémiologiques, l'analyse rapide d'un grand nombre d'échantillons dans des conditions de précision et de reproductibilité largement supérieures à celles de l'examen microscopique.

Dans deux autres applications, la détection de très faibles parasitémies peut, au contraire, être très importante. Ce sont les études de chloroquino-résistances et la prévention du paludisme post-transfusionnel. Pour cette prévention, le dépistage des crypto-porteurs de Plasmodium n'est que très imparfaitement réalisé par les techniques sérologiques qui révèlent souvent des titres séquellaires d'anticorps. Les sondes moléculaires, et surtout la PCR, pourraient apporter une solution rapide et sûre.

Par ailleurs, l'un des aspects essentiels de l'épidémiologie du paludisme concerne l'identification spécifique, chez l'anophèle vecteur, de sporozoïtes appartenant à une espèce plasmodiale donnée. Une solution partielle a été apportée par l'immunologie à l'aide d'anticorps monoclonaux ou polyclonaux. Les sondes moléculaires semblent susceptibles d'améliorer cette détermination si l'on se réfère aux résultats préliminaires obtenus à l'aide de sondes DNA ou avec des sondes moléculaires de RNA ribosomal (rRNA). Cependant la sensibilité de ce type de détection, de même que ses conditions pratiques d'emploi, doivent être encore améliorées.

La caractérisation de souches plasmodiales particulières constitue également l'un des chapitres les plus passionnants de la paludologie moderne. Indépendamment d'aspects théoriques, il s'agit, sur le plan pratique, d'identifier des souches possédant un pouvoir pathogène, des caractéristiques biologiques (production de protubérances ou knobs, gamétocytogenèse, par exemple), ou encore une chimio-sensibilité particulière. Des études comparatives inter-cépales ont été réalisées avec des oligonucléotides dérivés de la sonde originale de Franzen. Des profils de restriction différents ont été observés avec six souches de Plasmodium falciparum mais, avec ces sondes, il ne semble pas exister de corrélation nette avec la chloroquino-résistance. En effet, deux souches provenant des Philippines ont donné des profils de restriction identiques, alors que l'une de ces souches seulement était chloroquino-résistante. Au contraire, deux autres sondes (pBKR K1-14 et $753 \mathrm{BP}$ ) paraissent disposer d'une spécificité remarquable puisqu'elles permettraient de distinguer plusieurs isolats provenant d'une même souche. La sonde clonée à Grenoble (pUF 28) semble également capable, en Southern blot, de révéler différents profils de restriction pour des souches de diverses origines géographiques et de chloroquino-sensibilité inégales. Nos recherches se poursuivent dans cette voie, car un test permettant 
in vitro de rapidement étudier un grand nombre d'échantillons et d'en apprécier la chloroquino-sensibilité dans des conditions à la fois rapides et précises présenterait un intérêt pratique considérable.

Tous les travaux précédents se limitaient à Plasmodium falciparum. Pour les autres espèces, on a mis au point un ensemble de sondes de RNA ribosomal caractéristiques de $P$. falciparum, $P$. malariae, $P$. vivax et $P$. ovale. Enfin, l'emploi, comme sonde, d'un fragment génomique de DNA de Plasmodium berghei constitue une approche particulière. Cette sonde permet la détection des divers Plasmodium de l'homme mais avec une spécificité qui reste à définir et avec surtout une faible sensibilité (4 à 5000 Plasmodium/ échantillon de sang).

\section{Toxoplasmose}

Dans ce domaine, les premiers travaux sont récents (SAVVA, 1989). Grâce à une sonde DNA (pJMBG 37) radiomarquée; il est possible de déceler environ $20 \mathrm{ng}$ d'ADN cytoplasmique, ce qui correspond à environ $1,5 \times$ $10^{4}$ tachyzoïtes. Une sensibilité bien plus grande a été obtenue par la technique d'amplification génique in vitro (PCR) avec identification d'une séquence répétée 35 fois dans le génome toxoplasmique, le gène B1 (Burg et al., 1989). Dans un lysat cellulaire brut, il est possible avec cette sonde de déceler un seul parasite et, en partant d'ADN, le seuil de sensibilité est d'environ 10 toxoplasmes dans un échantillon contenant par ailleurs 100000 leucocytes humains, ce qui correspond au niveau maximum de réactions cellulaires dans une encéphalite toxoplasmique. Le gène B1 semble présent dans toutes les souches de $T$. gondii et il ne donne aucune réaction croisée avec d'autres micro-organismes susceptibles de localisation cérébrale chez les sujets immuno-déficients.

A Grenoble, à partir d'une sonde ADN (pTGR $1 \mathrm{E}$ comprenant $350 \mathrm{pb}$ ) clonée en 1989 (Cristina et al.), des oligonucléotides destinés à l'amplification génique in vitro ont été définis et synthétisés chimiquement. Après amplification, la sensibilité est de l'ordre de $20 \mathrm{fg}\left(10^{-15} \mathrm{~g}\right)$, ce qui correspond au $1 / 5^{\mathrm{e}}$ de la quantité d'ADN contenue dans un seul toxoplasme. La spécificité semble totale. La méthode est actuellement en cours d'optimisation pour le traitement de différents types d'échantillons biologiques en utilisation diagnostique de routine. Dans un avenir assez proche, il est donc possible que les sondes moléculaires ou les techniques dérivées constituent pour la toxoplasmose un moyen diagnostique particulièrement précieux. En effet, les autres procédés de mise en évidence de ce parasite sont longs, aléatoires ou mal commodes. Très largement utilisée, la sérologie présente des limites ou des incertitudes bien connues, dans les toxoplasmoses congénitales ou oculaires, notamment. Le problème est encore plus compliqué pour les toxoplasmoses cérébrales survenant chez des immuno-déprimés, Sidéens en particulier. L'évolution séro- logique de ces malades est en effet ininterprétable. L'atteinte encéphalitique correspond presque toujours à une décompensation de toxoplasmoses latentes mais nous ne disposons actuellement d'aucun élément prédictif permettant d'apprécier ce risque de décompensation et de mettre immédiatement en œuvre le traitement correspondant. L'analyse de divers prélèvements (sang, LCR) par sondes moléculaires ouvre donc des espoirs d'autant plus importants que la toxoplasmose semble devoir constituer l'une des principales causes de mortalité chez les Sidéens, avec la diminution relative des pneumocystoses sous l'effet des chimiopréventions primaires actuellement utilisées. Par ailleurs, pour Toxoplasma gondii comme pour de nombreux autres parasites, la notion d'un pouvoir pathogène propre à certaines souches a été plusieurs fois évoquée. Par isoenzymologie, il a été possible de typer des zymodèmes de souches particulières. Cette étude a pu être récemment reprise à Grenoble par l'étude de profils de restriction. Après fragmentation par action d'endonucléases, les DNA de 6 souches différentes de $T$. gondii ont été analysés par Southern blot en utilisant la sonde pTGR 1 E radiomarquée au ${ }^{32} \mathrm{P}$. Quatre types différents de profils de restriction ont été mis en évidence. Ils semblent corrélés avec le pouvoir pathogène mais il est cependant possible que d'autres paramètres tels que la production d'oocystes chez le chat ou celle de pseudokystes en culture cellulaire correspondent également à différents profils de restriction.

\section{Amibiase}

L'identification d'Entameba hystolitica dans un produit pathologique est souvent difficile et longue. Une sonde DNA de $145 \mathrm{pb}$ (pEH 5) permet de déceler environ 8 trophozoïtes par échantillon, sans risques de réaction croisée avec d'autres espèces amibiennes ou avec d'autres protozoaires intestinaux. Cette technique est probablement appelée à d'importants prolongements pratiques. Au moins aussi intéressantes sont les perspectives de typage de souches d'E. hystolitica présentant un pouvoir pathogène particulier. Cette notion repose sur des considérations épidémiologiques et cliniques déjà anciennes. Elle a pu être vérifiée en iso-enzymologie mais la découverte des modifications que les conditions de culture peuvent provoquer dans le zymodème d'une souche est venu compliquer la question. Récemment, une caractérisation cépale a été réalisée à l'aide de diverses sondes moléculaires (H6.6, R2.3, $\mathrm{R} 715$ et H16). Une corrélation a été retrouvée avec les profils iso-enzymatiques. Cependant, pour des zymodèmes pathogènes, deux groupes distincts de profils de restriction ont été identifiés, sans qu'aucune explication ne puisse être avancée actuellement. Par ailleurs, les profils de restriction sont également variables en fonction des conditions de culture. C'est ainsi que des souches voient simultanément modifiés leur zymodème et leur profil de restriction par passage de cultures xéniques à une multiplication axé- 
niques. L'origine de ce phénomène est encore discutée. Il peut s'agir soit de la sélection, au sein d'un isolat amibien, d'une sous-population pré-existante mais n'apparaissant que dans certaines conditions de culture, soit de séquences préexistantes dans le DNA génomique et qui se trouveraient amplifiées dans certaines conditions culturales.

\section{Trypanosomoses}

En ce qui concerne la Trypanosomose africaine, plusieurs espèces et sous-espèces ont pu être identifiées à l'aide de sondes DNA spécifiques. Ces résultats ouvrent de nouvelles perspectives aux études épidémiologiques, aussi bien chez l'homme que chez divers animaux. Ces études seront également facilitées par la possibilité d'identifier spécifiquement une espèce de trypanosome chez des glossines infectées. Une technique spéciale a même été proposée pour ce type d'étude sur le terrain (Touch blot). Plus récemment, la réaction d'amplification génique in vitro (PCR) a permis, après électrophorèse et coloration par le bromure d'éthidium, d'obtenir des résultats 100 fois plus sensibles qu'avec les sondes moléculaires. Il devient ainsi possible de détecter un seul trypanosome dans un prélèvement et même de suivre spécifiquement l'évolution de deux espèces ( $P$. brucei congolense, $P$. brucei rhodesiense), simultanément injectées à des souris.

L'étude de Trypanosoma cruzi, par sondes moléculaires, est facilitée par l'existence de séquences hautement répétées qui représentent environ $9 \%$ du DNA nucléaire total. Pour les trypanosomoses américaines, c'est dès 1984 qu'une sonde spécifique de $T$. cruzi a permis la détection d'environ 20 parasites $/ \mu \mathrm{l}$ de sang. Cette sensibilité a été par la suite améliorée, la spécificité étant totale vis-à-vis d'autres trypanosomes ou de leishmanies. La méthode a été appliquée à la mise en évidence de $T$. cruzi dans les déjections de Triatomes infestants et ceci en utilisant des sondes froides dont l'emploi sur le terrain est évidemment plus facile. Par ailleurs, à l'aide de cinq sondes provenant d'ADN kinétoplastique, on a pu caractériser 52 isolats et plusieurs clones de $T$. cruzi provenant d'hommes, de cobayes ou de triatomes infestés et isolés dans différentes régions d'Argentine ou du Chili.

\section{Leishmanioses}

La première sonde moléculaire a été clonée en 1982 à partir d'ADN nucléaire. Par la suite, les sondes utilisées provenaient d'ADN kinétoplastiques, l'ADN nucléaire présentant de trop grandes variations inter-cépales. L'ADN kinétoplastique permet, par l'étude des profils de restriction, de distinguer plusieurs espèces ou sous-espèces de Leishmania du nouveau monde. Les espèces de l'ancien monde (Leishmania infantum, L. major et L. donovani) ne sont pas encore différenciables par leurs profils de restriction. Certains fragments d'ADN kinétoplastique ont été clonés et utilisés comme sondes à des fins diagnostiques mais la sensibilité doit être encore améliorée. Il en est de même pour les sondes de L. mexicana, L. braziliensis, et L. tropica. Par la méthode du "Touch blot», il est possible de détecter directement des leishmanies à partir de prélèvement de lésions cutanées provoquées par L. mexicana. Avec des sondes froides marquées par le système biotine-streptavidine-phosphatase alcaline, le seuil de détection est d'environ 100 parasites par prélèvement. Par ailleurs, différents types de sondes ont été évidemment employées pour la détection des Leishmania chez des phlébotomes.

\section{Filarioses lymphatiques}

Les deux principales sondes oligo-nucléotidiques ( $\mathrm{pB}$ ma 68 et $\mathrm{pB}$ m 15 présentent d'importantes similitudes et appartiennent probablement à la même famille de séquences hautement répétées du génome de Brugia malayi. Après radiomarquage, ces deux sondes permettent la détection de 300 à $500 \mathrm{pg}$ de DNA de B. malayi, ce qui correspond à la quantité de DNA contenue dans une seule microfilaire. La spécificité et la sensibilité de ces sondes devraient donc permettre de modifier considérablement les conditions de dépistage des filarioses lymphatiques, et sur le plan épidémiologique, de préciser notamment si certaines filiaires animales comme $B$. pahangi peuvent également infester l'homme. De même, ces sondes permettent, dans un broyat de moustique infesté, la détection d'une seule larve infestante par hybridation in situ.

\section{Onchocercose}

Plusieurs sondes ont été préparées pour diverses onchocerques animales (O. armillata, O. gibsoni). Pour Onchocerca volvulus, certaines sondes $\mathrm{ADN}$ sont surtout spécifiques de genres (POV 26) alors que d'autres (pu Ovs 3) sont plus caractéristiques de l'espèce $O$. volvulus mais donnent diverses réactions croisées, par exemple avec $O$. ochengi, parasite des bovins. Cette sonde détecte moins de $100 \mathrm{pg}$ de DNA onchocerquien soit le $10^{\mathrm{e}}$ environ de DNA contenu dans une seule larve infestante. L’intérêt épidémiologique pour la détection de larves infestantes chez des simulies est cependant limité par les réactions croisées avec $O$. ochengi qui est présenté dans les mêmes aires de répartition géographique et peut être probablement transmise par les mêmes vecteurs. Plus récemment, des sondes plus spécifiques ont été isolées mais leur évaluation exige des études complémentaires.

Par ailleurs, l'un des problèmes essentiels de l'onchocercose est le pouvoir pathogène différent (en particulier sur le plan oculaire) d'Onchocerca volvulus en zone de forêt ou en zone de savane. Cette différence repose sur de nombreuses observations épidémiologiques. Elle est difficile à prouver par 
les techniques biologiques usuelles. Une sonde récemment isolée (pFS-1) semble capable de réaliser cette différenciation d'isolats provenants de diverses zones géographiques.

\section{Bilharzioses}

Les sondes moléculaires ont apporté ici plusieurs données nouvelles d'un intérêt épidémiologique considérable en épidémiologie. C'est ainsi que des sondes de DNA total de Shistosoma mansoni ou une sonde de 339 pb (p SMD 9) ont respectivement permis l'identification de mollusques infestés par diverses espèces de Schistosomes ou la caractérisation du sexe de cercaires. En fait, les résultats les plus complets ont été obtenus jusqu'ici à l'aide de trois sondes de RNA ribosomal de S. mansoni (p SM 389, p SM 889, p SM 890). L'étude des profils de restriction révélés par ces sondes a permis de distinguer entre elles différentes souches de $S$. mansoni ou de $S$. haematobium. Dans le groupe des schistosomes à œufs à éperon terminal, la technique permet de distinguer d'une part un ensemble de trois espèces ( $S$. intercalatum, $S$. bovis et $S$. curassoni) et, d'autre part, trois espèces individualisées (S. haematobium, S. matthei, et S. margrebowiei). En pathologie humaine, ces données sont d'autant plus intéressantes que, par exemple, $S$. matthei parasite habituel du bétail semble pouvoir parasiter l'homme dans certaines zones, en particulier au Transvaal. Par ailleurs, S. haematobium et $S$. mansoni peuvent, dans des infestations humaines mixtes, donner naissance à des hybrides décelables par ces sondes moléculaires. La même différenciation est possible pour des infestations mixtes à $S$. haematobium et à $S$. intercalatum. Enfin, l'infestation humaine par $S$. curassoni semble possible dans certaines zones du Sénégal mais était jusqu'ici très difficile à prouver. Il est possible, là aussi, que la solution soit apportée par les sondes moléculaires.

\section{Échinococcoses et cestodoses}

Deux espèces d'Echinococcus parasitent l'homme et provoquent chez lui des affections très différentes: Echinococcus granulosus et Echinococcus multilocularis. Il a été possible de les distinguer par Southern blot en utilisant trois sondes de rRNA de $S$. mansoni. Des résultats évidemment plus spécifiques ont été obtenus à partir de sondes DNA d'E. granulosus. La sonde pEG 18 permet en effet de distinguer au sein de l'espèce $E$. granulosus, des souches ou des isolats différents. Il s'agit-là d'une question extrêmement importante en hydatidologie, d'un point de vue épidémiologique, pathogénique, clinique, thérapeutique et surtout pronostique. On sait en effet que des souches apparemment identiques sur le plan morphologique et ne présentant que des différences iso-enzymatiques souvent difficiles à apprécier ont des hôtes intermédiaires différents (moutons et bovins d'une part, cheval, âne d'autre part) et chez l'homme un pouvoir pathogène très inégal (locali- sations préférentiellement hépatiques ou pulmonaires). Pour les souches « hépatiques ", il est d'ailleurs possible que certains kystes spontanément involutifs soient provoqués par des souches particulières que les sondes moléculaires pourraient permettre d'identifier plus aisément. Enfin, par hybridation in situ avec la sonde pEG 18 on peut détecter environ $0,15 \mathrm{pg}$ de DNA de E. granulosus. Ceci est donc applicable à l'identification spécifique des œufs du parasite chez le chien, ce qui peut présenter un intérêt épidémiologique considérable.

De même, des sondes hétérologues (rRNA de S. mansoni) ou homologues (DNA de Taenia solium) ont permis l'identification spécifique d'œufs de différentes espèces de Taeniidés ( $T$. solium, T. saginata, T. ovis, $T$. multiceps) mais, surtout, la distinction, au sein de l'espèce $T$. solium, de souches géographiquement distinctes et dont certaines peuvent présenter un pouvoir pathogène (cysticercose) ou une sensibilité au traitement particuliers.

$\mathrm{Au}$ total, les sondes moléculaires semblent donc ouvrir à la recherche parasitologique des perspectives extrêmement prometteuses. Certes, ce type de prévision a un caractère toujours aléatoire, et dans bien des domaines, des perspectives scientifiques qui paraissaient raisonnables ont été largement déçues. En s'appuyant sur les résultats actuellement acquis, les sondes moléculaires paraissent cependant capables d'apporter une solution élégante, rapide et précise à quelques-uns des principaux problèmes que l'on rencontre dans l'étude et dans le diagnostic des parasitoses. Elles peuvent notamment faciliter l'identification spécifique de parasites dont le diagnostic par d'autres techniques est difficile et qui, dans les suites d'immuno-déficiences acquises, ont pris ces dernières années une importance particulière en pathologie humaine (Pneumocystis carinii).

\section{RÉFÉRENCES}

Angelici M. C., Gramiccia M., Gradoni L. : Study on genetic polymorphism of Leishmania infantum through the analysis of restriction enzyme digestion patterns of kinetoplast DNA. Parasitology, 1989, 99, 301-309.

Barker D. C. : Molecular approaches to DNA diagnosis. Parasitology, 1989, 99, 125-146.

Burg J. L., Grover C. M., Pouletty P., Boothroyd J. C. : Direct and sensitive detection of a pathogenic Protozoan, Toxoplasma gondii, by Polymerase Chain Reaction. J. Microbiology, 1989, 27, 1787-1792.

Cristina N., Oury B., Ambroise-Thomas P., Santoro F. : DNA polymorphism and pathogenicity of Toxoplasma gondii strains. Parasit. Res. (submitted).

Garatte T., Harnett W., Parkhouse R. N. E. : Cloning of a speciesspecific DNA probe from Onchocerca gibsoni. Int. J. Parasitol., 1990, 20, 31-35.

Greig S., Ashall F., Hudson L. : Use of total Parasite DNA probes for the direct detection of Trypanosoma cruzi and Trypanosoma rangeli in domiciliary Rhodnius prolixus. Trans. $R$. Soc. Trop. Med. Hyg., 1990, 84, 59-60. 
Harnett. W., Chambers A. E., Renz A. P., Parkhouse R. N. E. : An oligonucleotide probe specific for Onchocerca volvulus. Mol. Biochem. Parasitol., 1989, 35, 119-125.

Lanar D. E., McLaughlin G. L., Zolg J. W., Wirth D. F., Chulay J. D. : Comparison of thick films, in vitro culture and DNA hybridization probes for detecting Plasmodium falciparum malaria. Am. J. Trop. Med. Hyg., 1989, 40, 3-6.

Moser D. R., Cook G. A., Ochs D. E., Bailey C. P., McKane M. R., Donelson J. E. : Detection of Trypanosoma congolense and Trypanosoma brucei species by DNA ampli- fication using the Polymerase Chain Reaction. Parasitology, 1989, 9, 57-66.

Oury B., Chumpitazi B., Cristina C., Markowicz Y., Mache R., Ambroise-Thomas P. : A new Plasmodium falciparum specific DNA probe for malaria. Mol. Biochem. Parasitol. (submitted).

Sava D. : Isolation of a potential DNA probe for Toxoplasma gondii strains. Microbios., 1989, 58, 165-172.

Waters A. D., McCutchan T. F. : Rapid, sensitive diagnosis of malaria based on ribosomal RNA. Lancet, 1989, $i$, 13431346. 